\title{
Semi-compatibility in Non-Archimedean Fuzzy Metric spaces
}

\author{
Seema Mehra \\ Department of Mathematics \\ M. D. University \\ Rohtak-124001(INDIA)
}

\author{
Renu Chugh \\ Department of Mathematics \\ M. D. University \\ Rohtak-124001(INDIA)
}

\begin{abstract}
The purpose of this paper is to prove a common fixed point theorem for six self maps using the concept of semicompatibility and weak compatibility of pair of self maps in Non- Archimedean fuzzy metric space.
\end{abstract}

\section{AMS SUBJECT CLASSIFICATION: 47H10, $54 \mathrm{H} 25$}

\section{KEYWORDS}

Semicompatible and weakly compatible mappings,

Non-Archimedean Fuzzy Metric Spaces.

\section{INTRODUCTION}

In [7], Istratescu initiated the study of fixed point theory in non-Archimedean Menger spaces and proved some fixed point theorems in this space using the same method as Cain and Kasriel in [5] for probabilistic metric space. The concept of fuzzy sets was coined by Zadeh [8] in his seminal paper in 1965. Since then, to use this concept in topology and analysis many authors have expansively developed the theory of fuzzy sets and application. Zadeh[9] anticipated that medical diagnosis would be the most likely application domain of Fuzzy set theory. The theory of fuzzy set is of fundamental importance in Medical diagnosis, effect of drugs, diagnosis process. Recently, a number of fixed point theorems and their applications in fuzzy set theory have been proved by several authors. George and Veeramani[1] and Kramosil and Michalek[6] have introduced the concept of fuzzy topological spaces induced by fuzzy metric which has very important applications in quantum particle physics particularly in connections with both string and $\varepsilon^{(\infty)}$ theory which were given and studied by El. Naschie [10- 12, 14].Many authors[2-3,13] have proved fixed point theorem in fuzzy(probabilistic) metric spaces. Recently, Dorel Mihet[4] proved a common fixed point theorem in Non- Archimedean fuzzy metric space. The purpose of this paper is to prove a common fixed point theorem for six self maps using the concept of semi-compatibility and weak compatibility of pair of self maps in Non- Archimedean fuzzy metric space.

\section{PREMINILARIES}

Definition 1.1 : A binary operation $*:[0,1] \times[0,1] \rightarrow[0,1]$ is a continuous tnorm

if it satisfies the following conditions:

(1) $*$ is associative and commutative,

(2) $*$ is continuous,

(3) $\mathrm{a} * 1=\mathrm{a}$ for all $\mathrm{a} \in[0,1]$,
(4) $\mathrm{a} * \mathrm{~b} \leq \mathrm{c} * \mathrm{~d}$ whenever $\mathrm{a} \leq \mathrm{c}$ and $\mathrm{b} \leq \mathrm{d}$, for each $a, b, c, d \in[0,1]$.

Example 1.1 : Two typical examples of continuous t-norm are $\mathrm{a} * \mathrm{~b}=\mathrm{ab}$ and $\mathrm{a} * \mathrm{~b}=\min (\mathrm{a}, \mathrm{b})$.

Definition 1.2[4]: The 3-tuple (X, M, *) is called a nonArchimedean fuzzy metric space (shortly, N.A. FM-space) if $\mathrm{X}$ is an arbitrary set, * is a continuous t norm and $\mathrm{M}$ is a fuzzy set in $X^{2} \times[0, \infty)$ satisfying the following conditions: For all $\mathrm{x}, \mathrm{y}, \mathrm{z} \in \mathrm{X}$ and $\mathrm{s}, \mathrm{t}>0$,

(NFM-1) $M(x, y, 0)=0$,

$(N F M-2) M(x, y, t)=1$, for all $t>0$ if and only if $x=y$, (NFM-3) $M(x, y, t)=M(y, x, t)$,

(NFM-4) M(x, y, t ) $* M(y, z, s) \leq M(x, z, \max \{t, s\})$

Or equivalently $\mathrm{M}(\mathrm{x}, \mathrm{y}, \mathrm{t}) * \mathrm{M}(\mathrm{y}, \mathrm{z}, \mathrm{t}) \leq \mathrm{M}(\mathrm{x}, \mathrm{z}, \mathrm{t})$

(NFM-5) $\mathrm{M}(\mathrm{x}, \mathrm{y},):.[0, \infty) \rightarrow[0,1]$ is left continuous. Definition 1.3 :For $t \in(0, \infty)$, we define the closed ball $\mathrm{B}[\mathrm{x}, \mathrm{r}, \mathrm{t}]$ with centre $\mathrm{x} \in \mathrm{X}$ and radius $\mathrm{r} \in(0,1)$ as

$B[x, r, t]=\{y \in X, M(x, y, t)>1-r\}$.

Definition 1.4 : A N.A. FM-space (X, M, *) is said to be of type (C)g if there exists a $g \in \Omega$ such that $\mathrm{g}(\mathrm{M}(\mathrm{x}, \mathrm{y}, \mathrm{t})) \leq \mathrm{g}(\mathrm{M}(\mathrm{x}, \mathrm{z}, \mathrm{t}))+\mathrm{g}(\mathrm{M}(\mathrm{z}, \mathrm{y}, \mathrm{t}))$ for all $\mathrm{x}, \mathrm{y}, \mathrm{z} \in \mathrm{X}$ and $\mathrm{t} \geq 0$, where $\Omega=\{\mathrm{g}: \mathrm{g}:[0,1] \rightarrow[0, \infty)$ is continuous, strictly decreasing, $\mathrm{g}(1)=0$ and $\mathrm{g}(0)<\infty\}$.

Definition 1.5: A N. A. FM-space (X, M, * ) is said to be of type (D)g if there exists a $g \in \Omega$ such that $\mathrm{g}(*(\mathrm{~s}, \mathrm{t})) \leq \mathrm{g}(\mathrm{s})+\mathrm{g}(\mathrm{t})$ for all $\mathrm{s}, \mathrm{t} \in[0,1]$.

Definition 1.6: A pair of maps A and B is called weakly compatible pair if they commute at coincidence points i.e., $\mathrm{Ax}=\mathrm{Bx}$ if and only if $\mathrm{ABx}=\mathrm{BAx}$.

Definition 1.7: Let $A$ and $S$ be mappings from N.A. FMspace $(\mathrm{X}, \mathrm{M}, *)$ in to itself. The mappings $\mathrm{A}$ and $\mathrm{S}$ are said to be compatible if $\lim _{n \rightarrow \infty} \mathrm{g}\left(\mathrm{M}\left(\mathrm{ASx}_{\mathrm{n}}, \mathrm{SAx}_{\mathrm{n}}, \mathrm{t}\right)\right)=0$,

for all $t>0$, whenever $\left\{x_{n}\right\}$ is a sequence in $X$ such that

$\lim _{\mathrm{n} \rightarrow \infty} \mathrm{Ax}_{\mathrm{n}}=\lim _{\mathrm{n} \rightarrow \infty} \mathrm{Sx}_{\mathrm{n}}=\mathrm{z}$ for some $\mathrm{z} \in \mathrm{X}$.

Definition 1.8 : Let A, S : X $\rightarrow$ X be mappings. The pair

$(\mathrm{A}, \mathrm{S})$ is said to be weak-compatible if they commute at their coincidence points, i.e., if $\mathrm{Az}=\mathrm{Sz}$ for some $\mathrm{w} \in \mathrm{X}$ then such that $\mathrm{ASw}=\mathrm{SAw}$. 
Definition 1.9 : Let A and S be mappings from an N.A. FMspace $(\mathrm{X}, \mathrm{M}, *)$ into itself. Then the mappings are said to be semi-compatible if

$\lim _{n \rightarrow \infty} g\left(M\left(A S x_{n}, S x, t\right)\right)=0$, for all $t>0$, whenever $\left\{x_{n}\right\}$ is a sequence in $\mathrm{X}$ such that $\lim _{\mathrm{n} \rightarrow \infty} \mathrm{Ax}_{\mathrm{n}}=\lim _{\mathrm{n} \rightarrow \infty} \mathrm{Sx}_{\mathrm{n}}=\mathrm{x}$ for some $\mathrm{x} \in \mathrm{X}$.

Proposition 1.1: Let $A$ and $S$ be self-maps on an N.A. FMspace $(X, M, *)$. If $S$ is continuous, then $(A, S)$ is semicompatible if and only if (A, S) is compatible.

Proof: Consider a sequence $\left\{x_{n}\right\}$ in $X$ such that $\{A x n\}$ and $\left\{\mathrm{Sx}_{\mathrm{n}}\right\}$ converges to $\mathrm{u} \in \mathrm{X}$. Suppose that $(\mathrm{A}, \mathrm{S})$ is compatible, then

$\mathrm{g}\left(\mathrm{M}\left(\mathrm{ASx}_{\mathrm{n}}, \mathrm{Su}, \mathrm{t}\right)\right) \leq \mathrm{g}\left(\mathrm{M}\left(\mathrm{ASx}_{\mathrm{n}}, \mathrm{SAx}_{\mathrm{n}}, \mathrm{t}\right)\right)+\mathrm{g}\left(\mathrm{M}\left(\mathrm{SAx}_{\mathrm{n}}, \mathrm{Su} \mathrm{t}\right)\right)$ letting $\mathrm{n} \rightarrow \infty$, Since (A, S) is compatible, we have $\lim _{n \rightarrow \infty} \mathrm{g}\left(\mathrm{M}\left(\mathrm{ASx} x_{\mathrm{n}}, \mathrm{Su}, \mathrm{t}\right)\right)=0$. Hence $\mathrm{ASx}_{\mathrm{n}} \rightarrow S u$,i.e., $(\mathrm{A}, \mathrm{S})$ is semi-compatible.

Conversely, Suppose that $(\mathrm{A}, \mathrm{S})$ is semi-compatible, then $\mathrm{g}\left(\mathrm{M}\left(\mathrm{ASx}_{\mathrm{n}}, \mathrm{SAx}_{\mathrm{n}}, \mathrm{t}\right)\right) \leq \mathrm{g}\left(\mathrm{M}\left(\mathrm{ASx}_{\mathrm{n}}, \mathrm{Su}, \mathrm{t}\right)\right)+\mathrm{g}\left(\mathrm{M}\left(\mathrm{SAx}_{\mathrm{n}}, \mathrm{Su} \mathrm{t}\right)\right)$. letting $\mathrm{n} \rightarrow \infty$, Since $(\mathrm{A}, \mathrm{S})$ is semi-compatible and $\mathrm{S}$ is continuous, we have

SAxn $\rightarrow S u$ and this implies

$\lim _{\mathrm{n} \rightarrow \infty} \mathrm{g}\left(\mathrm{M}\left(\mathrm{ASx} \mathrm{x}_{\mathrm{n}}, \mathrm{Su}, \mathrm{t}\right)\right)=0$.

Hence the pair $(\mathrm{A}, \mathrm{S})$ is compatible.

The following is an example of a pair of self-maps (A, S) which is compatible but not semi-compatible. Further, it is also seen here that the semi-compatibility of the pair (A, S) need not imply the semi-compatibility of (S,A).

Example 1.2. Let $X=[0,1]$ and let $(X, M, *)$ be the N.A. FM-space with

$g(M(x, y, t))=g\left[\exp \frac{|x-y|}{t}\right]^{-1}$ for all $x, y \in X, t>0$.

Define self-map S as follows:

$$
\left.S x=\begin{array}{cc}
x \text { if } & 0 \leq x \prec \frac{1}{2} \\
1 \text { if } & x \geq \frac{1}{2}
\end{array}\right\}
$$

Let $\mathrm{I}$ be the identity map on $\mathrm{X}$ and $x_{n}=\frac{1}{2}-\frac{1}{n}$. Then, $\left\{\mathrm{Ix}_{\mathrm{n}}\right\}=\left\{\mathrm{x}_{\mathrm{n}}\right\} \rightarrow \frac{1}{2}$

And $\left\{\mathrm{Sx}_{\mathrm{n}}\right\}=\left\{\mathrm{x}_{\mathrm{n}}\right\} \rightarrow \frac{1}{2}$. Thus $\left\{\mathrm{ISx}_{\mathrm{n}}\right\}=$ $\left\{\mathrm{Sx}_{\mathrm{n}}\right\} \rightarrow \frac{1}{2} \neq \mathrm{S}\left(\frac{1}{2}\right)$. Hence (I, S) is not semicompatible.

Again as (I, S) is commuting, it is compatible. Futher, for any sequence $\left\{x_{n}\right\}$ in $X$ such that $\left\{x_{n}\right\} \rightarrow x$, we have $\left\{\operatorname{SIx}_{n}\right\}=$ $\left\{\mathrm{Sx}_{\mathrm{n}}\right\} \rightarrow \mathrm{x}=\mathrm{Ix}$. Hence $(\mathrm{S}, \mathrm{I})$ is always semicompatible.
Remark 1.1. The above example gives an important aspect of semicompatibility as the pair of self-maps (I,S) is commuting, hence it is weakly commuting, compatible and weak compatible yet it is not semicompatible. Further, it is to be noted that the pair (S, I) is semicompatible but (I,S) is not semicompatible here.

The following is an example of a pair of self-maps (A, S) which is semicompatible but not compatible.

Example 1.2 : Let $(\mathrm{X}, \mathrm{M}, *)$ be the N.A. FM-space, where $\mathrm{X}=[0,2]$, with $\mathrm{t}$-norm defined by $\mathrm{a} * \mathrm{~b}=\min \{\mathrm{a}, \mathrm{b}\}$, for all $\mathrm{a}$, $\mathrm{b} \in[0,1]$ and

$\mathrm{g}(\mathrm{M}(\mathrm{x}, \mathrm{y}, \mathrm{t}))=\mathrm{g}\left(\frac{t}{t+d(x, y)}\right)$ for all $\mathrm{t}>0$ and

$\mathrm{g}(\mathrm{M}(\mathrm{x}, \mathrm{y}, 0))<\infty$, for all $\mathrm{x}, \mathrm{y} \in \mathrm{X}$. Define self-maps A and $\mathrm{S}$ on $\mathrm{X}$ follows :

$\mathrm{Ax}=\left\{\begin{array}{l}2 \text { if } 0 \leq x \leq 1 \\ \frac{x}{2} \text { if } 1 \prec x \leq \frac{1}{2}\end{array}, \mathrm{Sx}=\left\{\begin{array}{c}2 \text { if } x=1 \\ \frac{x+3}{5} \text { otherwise }\end{array}\right.\right.$

And $x_{n}=2-\frac{1}{2 n}$. Then we have $\mathrm{S}(1)=\mathrm{A}(1)=2$ and $\mathrm{S}(2)$ $=\mathrm{A}(2)=1$. Also $\mathrm{SA}(1)=\mathrm{AS}(1)=1$ and $\mathrm{SA}(2)=\mathrm{AS}(2)=2$. Thus (A, S) is weak compatible. Again,

$\mathrm{Ax}_{\mathrm{n}}=1-\frac{1}{4 n}, \mathrm{Sx}_{\mathrm{n}}=1-\frac{1}{10 n}$

Thus, $A x_{n} \rightarrow 1, S x_{n} \rightarrow 1$.

Hence $\mathrm{u}=1$

Further, $\mathrm{SAx}_{\mathrm{n}}=\frac{4}{5}-\frac{1}{20 n}, \mathrm{ASx}_{\mathrm{n}}=2$.

Now,

$\lim _{\mathrm{n} \rightarrow \infty} \mathrm{g}\left(\mathrm{M}\left(\mathrm{ASx} \mathrm{x}_{\mathrm{n}}, \mathrm{Su}, \mathrm{t}\right)\right)=\mathrm{g}(\mathrm{M}(2,2, \mathrm{t}))=0$, 
$\lim _{n \rightarrow \infty} g\left(M\left(A S x_{n}, S A x_{n}, t\right)\right)=\lim _{n \rightarrow \infty} g\left(M\left(2, \frac{4}{5}-\frac{1}{20 n}, t\right)\right)$

$=\mathrm{g}\left(\frac{t}{t+\frac{6}{5}}\right) \neq 0$, for all $\mathrm{t}>0$.Hence $(\mathrm{A}, \mathrm{S})$ is

semicompatible but it is not compatible.

Remark 1.2. (1) If a N.A.FM-space (X,M, *) is of type (D)g then $(X, M, *)$ is of type $(C) g$.

(2) If $(\mathrm{X}, \mathrm{M}, *)$ is a N.A. FM-space and $* \geq * \mathrm{~m}$, where $* \mathrm{~m}(\mathrm{~s}, \mathrm{t})=\max \{\mathrm{s}+\mathrm{t}-1,0\}$, then $\left(\mathrm{X}, \mathrm{M},{ }^{*}\right)$ is of type (D)g for $\mathrm{g} \in \Omega$ defined by $\mathrm{g}(\mathrm{t})=1-\mathrm{t}$.

Throughout this paper, let $(\mathrm{X}, \mathrm{M}, *)$ be a complete N.A.FMspace of type (D)g with a continuous strictly increasing tnorm D .

Let $\phi:[0,+\infty) \rightarrow[0,+\infty)$ be a function satisfying the following condition $(\Phi)$ :

(Ф) $\phi$ is upper-semicontinuous from the right and $\phi(t)<t$ for all $\mathrm{t}>0$.

Lemma 1.1. [16] If a function $\phi:[0,+\infty) \rightarrow[0,+\infty)$ satisfies the condition $(\Phi)$, then we have

(1) For all $t \geq 0, \lim _{n \rightarrow \infty} \phi^{n}(t)=0$, where $\phi^{n}(t)$ is the $n$-th iteration of $\phi(t)$.

(2) If $\left\{t_{n}\right\}$ is a non-decreasing sequence of real numbers and $\mathrm{t}_{\mathrm{n}+1} \leq \phi\left(\mathrm{t}_{\mathrm{n}}\right), \quad \mathrm{n}=1,2, \ldots$, then $\lim _{\mathrm{n} \rightarrow \infty} \mathrm{t}_{\mathrm{n}}=0$. In particular, if $\mathrm{t} \leq \phi(\mathrm{t})$ for all $\mathrm{t} \geq 0$, then $\mathrm{t}=0$.

Lemma 1.2[15] Let $\left\{y_{n}\right\}$ be a sequence in $X$ such that $\lim _{n \rightarrow \infty}$

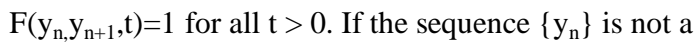
Cauchy sequence in $\mathrm{X}$, then there exist $\in_{0}>0, \mathrm{t}_{0}>0$, two sequences $\left\{\mathrm{m}_{\mathrm{i}}\right\},\left\{\mathrm{n}_{\mathrm{i}}\right\}$ of positive integers such that

(1) $\mathrm{m}_{\mathrm{i}}>\mathrm{n}_{\mathrm{i}}+1$, and $\mathrm{n}_{\mathrm{i}} \rightarrow \infty$ as $\mathrm{i} \rightarrow \infty$,

(2) $\mathrm{F}\left(\mathrm{y}_{m_{i}}, \mathrm{y}_{n_{i}}, \mathrm{t}_{0}\right)<1-\epsilon_{0}$ and $\mathrm{F}\left(\mathrm{y}_{m_{i}-1}, \mathrm{y}_{n_{i}}, \mathrm{t}_{0}\right) \geq 1-\epsilon_{0}, \mathrm{i}=$ $1,2, \ldots$

\section{RESULTS}

Theorem 1 : Let A, B, S , T, P and Q :X $\rightarrow X$ be mappings such that

(1) $\mathrm{P}(\mathrm{X}) \subset \mathrm{ST}(\mathrm{X})$ and $\mathrm{Q}(\mathrm{X}) \subset \mathrm{AB}(\mathrm{X})$,

(2) $\mathrm{AB}=\mathrm{BA}, \mathrm{ST}=\mathrm{TS}, \mathrm{PB}=\mathrm{BP}, \mathrm{QS}=\mathrm{SQ}, \mathrm{QT}=\mathrm{TQ}$,

(3) $\mathrm{g}(\mathrm{M}(\mathrm{Px}, \mathrm{Qy}, \mathrm{t})) \leq \phi(\max \{\mathrm{g}(\mathrm{M}(\mathrm{ABx}, \mathrm{Ty}, \mathrm{t}))$,

$(\mathrm{M}(\mathrm{ABx}, \mathrm{Px}, \mathrm{t})), \mathrm{g}(\mathrm{M}(\mathrm{STy}, \mathrm{Qy}, \mathrm{t}))$,

$1 / 2(\mathrm{~g}(\mathrm{M}(\mathrm{ABx}, \mathrm{Qy}, \mathrm{t}))+\mathrm{g}(\mathrm{M}(\mathrm{STy}, \mathrm{Px}, \mathrm{t})))\})$ for all $\mathrm{t}>0$,

where a function $\phi:[0, \infty) \rightarrow[0, \infty)$ satisfies the condition $(\Phi)$.

(4) the pair $(\mathrm{P}, \mathrm{AB})$ is semicompatible and $(\mathrm{Q}, \mathrm{ST})$ is weakly compatible

(5) Either $\mathrm{AB}$ or $\mathrm{P}$ is continuous

Then A, B, S, T, P and Q have a unique common fixed point in $\mathrm{X}$.
Proof. Let $\mathrm{x}_{0} \in \mathrm{X}$, then by (1), there exists a point $\mathrm{x}_{1} \in \mathrm{X}$ such that $\mathrm{Px}_{0}=\mathrm{STx}_{1}$ since $\mathrm{P}(\mathrm{X}) \subset \mathrm{ST}(\mathrm{X})$. Since $\mathrm{Q}(\mathrm{X}) \subset$ $\mathrm{AB}(\mathrm{X})$, for this point $\mathrm{x}_{1}$, we can choose a point $\mathrm{x}_{2} \in \mathrm{X}$ such that $\mathrm{Qx}_{1}=\mathrm{ABx}_{2}$ and so on. Inductively, we can define a sequence $\left\{y_{n}\right\}$ in $X$ such that

(6) $\mathrm{y}_{2 \mathrm{n}}=\mathrm{Px}_{2 \mathrm{n}}=\mathrm{STx}_{2 \mathrm{n}+1}$ and $\mathrm{y}_{2 \mathrm{n}+1}=\mathrm{ABx}_{2 \mathrm{n}+2}=\mathrm{Qx}_{2 \mathrm{n}+1}$ for $\mathrm{n}$ $=0,1,2 \ldots$, Now we prove the sequence $\left\{y_{n}\right\}$, defined by $(6)$, such that

$\lim _{n \rightarrow \infty} g\left(M\left(y_{n}, y_{n+1}, t\right)\right)=0$ for all $t>0$ is a Cauchy sequence in $\mathrm{X}$.

Since $g \in \Omega$, it follows that $\lim _{\mathrm{n} \rightarrow \infty} M\left(\mathrm{y}_{\mathrm{n}}, \mathrm{y}_{\mathrm{n}+1}, \mathrm{t}\right)=1$ for all $t>0$ if and only if $\lim _{n \rightarrow \infty} g\left(M\left(y_{n}, y_{n+1}, t\right)\right)=0$ for all $t>0$. By Lemma 1.2. if $\left\{y_{n}\right\}$ is not a Cauchy sequence in $X$, then there exist $\epsilon_{0}>0, \mathrm{t}_{0}>0$ and two sequences $\left\{\mathrm{m}_{\mathrm{i}}\right\},\left\{\mathrm{n}_{\mathrm{i}}\right\}$ of positive integers such that

(A) $\mathrm{m}_{\mathrm{i}}>\mathrm{n}_{\mathrm{i}}+1$, and $\mathrm{n}_{\mathrm{i}} \rightarrow \infty$ as i $\rightarrow \infty$,

(B) $\mathrm{F}\left(\mathrm{y}_{m_{i}}, \mathrm{y}_{n_{i}}, \mathrm{t}_{0}\right)>1-\epsilon_{0}$ and $\mathrm{F}\left(\mathrm{y}_{m_{i}-1}, \mathrm{y}_{n_{i}}, \mathrm{t}_{0}\right) \leq 1-\epsilon_{0}, \mathrm{i}=$ $1,2, \ldots$

Thus we have

$\mathrm{g}\left(1-\in_{0}\right)<\mathrm{g}\left(\mathrm{M}\left(\mathrm{y}_{m_{i}}, \mathrm{y}_{n_{i}}, \mathrm{t}_{0}\right)\right)$

(7) $\leq \mathrm{g}\left(\mathrm{M}\left(y_{m_{i}}, y_{m_{i}+1}, \mathrm{t}_{0}\right)\right)+\mathrm{g}\left(\mathrm{M}\left(y_{m_{i}+1}, \mathrm{y}_{n_{i}}, \mathrm{t}_{0}\right)\right)$

$$
\leq \mathrm{g}\left(\mathrm{M}\left(y_{m_{i}}, y_{m_{i}+1}, \mathrm{t}_{0}\right)\right)+\mathrm{g}\left(1-\varepsilon_{0}\right)
$$

Letting $\mathrm{i} \rightarrow \infty$ in (7), we have

(8) $\lim _{\mathrm{n} \rightarrow \infty} \mathrm{g}\left(\mathrm{M}\left(\mathrm{y}_{m_{i}}, \mathrm{y}_{n_{i}}, \mathrm{t}_{0}\right)\right)=\mathrm{g}\left(1-\epsilon_{0}\right)$.

On the other hand, we have

$$
\mathrm{g}\left(1-\epsilon_{0}\right)<\mathrm{g}\left(\mathrm{M}\left(\mathrm{y}_{m_{i}}, \mathrm{y}_{n_{i}}, \mathrm{t}_{0}\right)\right)
$$

$$
\leq \mathrm{g}\left(\mathrm{M}\left(\mathrm{y}_{n_{i}}, \mathrm{y}_{n_{i}+1}, \mathrm{t}_{0}\right)\right)+\mathrm{g}\left(\mathrm{M}\left(\mathrm{y}_{n_{i}+1}, y_{m_{i}}, \mathrm{t}_{0}\right)\right)
$$

Now, consider $\mathrm{g}\left(\mathrm{M}\left(\mathrm{y}_{n_{i}+1}, y_{m_{i}}, \mathrm{t}_{0}\right)\right)$ in (9). Without loss of generality assume that both $\mathrm{n}_{\mathrm{i}}$ and $\mathrm{m}_{\mathrm{i}}$ are even.

Then , by (3), we have

$$
\begin{aligned}
& \mathrm{g}\left(\mathrm{M}\left(\mathrm{y}_{n_{i}+1}, y_{m_{i}}, \mathrm{t}_{0}\right)\right)=\mathrm{g}\left(\mathrm{M}\left(\mathrm{Px}_{m_{i}}, \mathrm{Qx} \mathrm{n}_{n_{i}+1}, \mathrm{t}_{0}\right)\right) \\
& \leq \phi\left(\operatorname { m a x } \left\{\mathrm{g}\left(\mathrm{M}\left(\mathrm{ABx}_{m_{i}}, \mathrm{STx}_{n_{i}+1}, \mathrm{t}_{0}\right)\right),\right.\right. \\
& \mathrm{g}\left(\mathrm{M}\left(\mathrm{ABx}_{m_{i}}, \mathrm{Px}_{m_{i}}, \mathrm{t}_{0}\right)\right), \mathrm{g}\left(\mathrm{M}\left(\mathrm{STx}_{n_{i}+1}, \mathrm{Qx}_{n_{i}+1}, \mathrm{t}_{0}\right)\right) \text {, } \\
& \left.\left.1 / 2\left(\mathrm{~g}\left(\mathrm{M}\left(\mathrm{ABx}_{m_{i}}, \mathrm{Qx}_{n_{i}+1}, \mathrm{t}_{0}\right)\right)+\mathrm{g}\left(\mathrm{M}\left(\mathrm{STx}_{n_{i}+1}, \mathrm{Px}_{m_{i}}, \mathrm{t}_{0}\right)\right)\right)\right\}\right) \\
& \text { (10) } \quad=\phi\left(\operatorname { m a x } \left\{\mathrm{g}\left(\mathrm{M}\left(\mathrm{y}_{m_{i}-1} \mathrm{y}_{n_{i}} \mathrm{n}_{\mathrm{i}}, \mathrm{t}_{0}\right)\right)\right.\right. \text {, } \\
& \mathrm{g}\left(\mathrm{M}\left(\mathrm{y}_{m_{i}-1}, \mathrm{y}_{m_{i}}, \mathrm{t}_{0}\right)\right), \mathrm{g}\left(\mathrm{M}\left(\mathrm{y}_{n_{i}}, \mathrm{y}_{n_{i}+1}, \mathrm{t}_{0}\right)\right), \\
& \left.\left.1 / 2\left(\mathrm{~g}\left(\mathrm{M}\left(\mathrm{y}_{m_{i}-1}, \mathrm{y}_{n_{i}+1}, \mathrm{t}_{0}\right)\right)+\mathrm{g}\left(\mathrm{M}\left(\mathrm{y}_{n_{i}}, \mathrm{y}_{m_{i}}, \mathrm{t}_{0}\right)\right)\right)\right\}\right)
\end{aligned}
$$


By (8), (9) and (10), letting $i \rightarrow \infty$ in (10), we have

$$
\begin{aligned}
\mathrm{g}\left(1-\in_{0}\right) & \leq \phi\left(\max \left\{\mathrm{g}\left(1-\in_{0}\right), 0,0, \mathrm{~g}\left(1-\in_{0}\right)\right\}\right) \\
& =\phi\left(\mathrm{g}\left(1-\in_{0}\right)\right)<\mathrm{g}\left(1-\in_{0}\right)
\end{aligned}
$$

which is a contradiction. Therefore, $\left\{\mathrm{y}_{\mathrm{n}}\right\}$ is a Cauchysequence in $\mathrm{X}$.

Now, we prove $\lim _{n \rightarrow \infty} g\left(M\left(y_{n}, y_{n+1}, t_{0}\right)\right)=0$ for all $t>0$. In fact, by (3) and (4), we have

$$
\begin{aligned}
& \mathrm{g}\left(\mathrm{M}\left(\mathrm{y}_{2 \mathrm{n}}, \mathrm{y}_{2 \mathrm{n}+1}, \mathrm{t}\right)\right)=\mathrm{g}\left(\mathrm{M}\left(\mathrm{Px}_{2 \mathrm{n}}, \mathrm{Qx}_{2 \mathrm{n}+1}, \mathrm{t}\right)\right) \\
& \leq \phi\left(\operatorname { m a x } \left\{\mathrm{g}\left(\mathrm{M}\left(\mathrm{ABx}_{2 \mathrm{n}}, \mathrm{STx}_{2 \mathrm{n}+1}, \mathrm{t}\right)\right),\right.\right. \\
& \mathrm{g}\left(\mathrm{M}\left(\mathrm{ABx}_{2 \mathrm{n}}, \mathrm{Px}_{2 \mathrm{n}}, \mathrm{t}\right)\right), \mathrm{g}\left(\mathrm{M}\left(\mathrm{STx}_{2 \mathrm{n}+1}, \mathrm{Qx}_{2 \mathrm{n}+1}, \mathrm{t}\right)\right), \\
& \left.\left.1 / 2\left(\mathrm{~g}\left(\mathrm{M}\left(\mathrm{ABx}_{2 \mathrm{n}}, \mathrm{Qx}_{2 \mathrm{n}+1}, \mathrm{t}\right)\right)+\mathrm{g}\left(\mathrm{M}\left(\mathrm{STx}_{2 \mathrm{n}+1}, \mathrm{Px}_{2 \mathrm{n}}, \mathrm{t}\right)\right)\right)\right\}\right) \\
& =\phi\left(\operatorname { m a x } \left\{g\left(M\left(y_{2 n-1}, y_{2 n}, t\right)\right), g\left(M\left(y_{2 n-1}, y_{2 n}, t\right)\right),\right.\right. \\
& \left.\left.\left.\mathrm{g}\left(\mathrm{M}\left(\mathrm{y}_{2 \mathrm{n}}, \mathrm{y}_{2 \mathrm{n}+1}, \mathrm{t}\right)\right), 1 / 2\left(\mathrm{~g}\left(\mathrm{M}\left(\mathrm{y}_{2 \mathrm{n}-1}, \mathrm{y}_{2 \mathrm{n}+1}, \mathrm{t}\right)\right)+\mathrm{g}(1)\right)\right)\right\}\right)
\end{aligned}
$$

$\leq \phi\left(\max \left\{\mathrm{g}\left(\mathrm{M}\left(\mathrm{y}_{2 \mathrm{n}-1}, \mathrm{y}_{2 \mathrm{n}}, \mathrm{t}\right)\right), \mathrm{g}\left(\mathrm{M}\left(\mathrm{y}_{2 \mathrm{n}}, \mathrm{y}_{2 \mathrm{n}+1}, \mathrm{t}\right)\right)\right.\right.$,

$$
\left.\left.1 / 2\left(\mathrm{~g}\left(\mathrm{M}\left(\mathrm{y}_{2 \mathrm{n}-1}, \mathrm{y}_{2 \mathrm{n}}, \mathrm{t}\right)\right)+\mathrm{g}\left(\mathrm{M}\left(\mathrm{y}_{2 \mathrm{n}}, \mathrm{y}_{2 \mathrm{n}+1}, \mathrm{t}\right)\right)\right)\right\}\right) .
$$

If $\mathrm{g}\left(\mathrm{M}\left(\mathrm{y}_{2 \mathrm{n}-1}, \mathrm{y}_{2 \mathrm{n}}, \mathrm{t}\right)\right) \leq \mathrm{g}\left(\mathrm{M}\left(\mathrm{y}_{2 \mathrm{n}}, \mathrm{y}_{2 \mathrm{n}+1}, \mathrm{t}\right)\right)$ for all $\mathrm{t}>0$, then, by (3),

$\mathrm{g}\left(\mathrm{M}\left(\mathrm{y}_{2 \mathrm{n}}, \mathrm{y}_{2 \mathrm{n}+1}, \mathrm{t}\right)\right) \leq \phi\left(\mathrm{g}\left(\mathrm{M}\left(\mathrm{y}_{2 \mathrm{n}}, \mathrm{y}_{2 \mathrm{n}+1}, \mathrm{t}\right)\right)\right)$ which means that by Lemma 1.1.,

$g\left(M\left(y_{2 n}, y_{2 n+1}, t\right)\right)=0$ for all $t>0$. Similarly, we have $\mathrm{g}\left(\mathrm{M}\left(\mathrm{y}_{2 \mathrm{n}+1}, \mathrm{y}_{2 \mathrm{n}+2}, \mathrm{t}\right)\right)=0$ for all $\mathrm{t}>0$. Thus we have

$\lim _{n \rightarrow \infty} g\left(M\left(y_{n} y_{n+1}, t\right)\right)=0$ for all $t>0$. On the other hand, if

$g\left(M\left(y_{2 n-1}, y_{2 n}, t\right)\right) \geq g\left(M\left(y_{2 n}, y_{2 n+1}, t\right)\right)$, then by (3), we have $\mathrm{g}\left(\mathrm{M}\left(\mathrm{y}_{2 \mathrm{n}}, \mathrm{y}_{2 \mathrm{n}+1}, \mathrm{t}\right)\right) \leq \phi\left(\mathrm{g}\left(\mathrm{M}\left(\mathrm{y}_{2 \mathrm{n}-1}, \mathrm{y}_{2 \mathrm{n}}, \mathrm{t}\right)\right)\right.$ for all $\mathrm{t}>0$.

Similarly, $g\left(M\left(y_{2 n+1}, y_{2 n+2}, t\right)\right) \leq \phi\left(g\left(M\left(y_{2 n}, y_{2 n+1}, t\right)\right)\right)$, for all $\mathrm{t}>0$. Thus we have

$\mathrm{g}\left(\mathrm{M}\left(\mathrm{y}_{\mathrm{n}}, \mathrm{y}_{\mathrm{n}+1}, \mathrm{t}\right)\right) \leq \phi\left(\mathrm{g}\left(\mathrm{M}\left(\mathrm{y}_{\mathrm{n}-1}, \mathrm{y}_{\mathrm{n}}, \mathrm{t}\right)\right)\right.$ for all $\mathrm{t}>0$ and $\mathrm{n}=1,2,3, \ldots$ Therefore by Lemma 1.1 ,

$\lim _{n \rightarrow \infty} g\left(M\left(y_{n}, y_{n+1}, t\right)\right)=0$ for all $t>0$, which implies that $\left\{y_{n}\right\}$ is a Cauchy sequence in $X$ by Lemma 1.2. Since $\left(\mathrm{X}, \mathrm{M},{ }^{*}\right)$ is complete, the sequence $\left\{\mathrm{y}_{\mathrm{n}}\right\}$ converges to a point $\mathrm{z} \in \mathrm{X}$ and so the subsequences $\left\{\mathrm{Px}_{2 \mathrm{n}}\right\},\left\{\mathrm{Qx}_{2 \mathrm{n}+1}\right\}$, $\left\{\mathrm{ABx}_{2 \mathrm{n}}\right)$ and $\left\{\mathrm{STx}_{2 \mathrm{n}+1}\right\}$ of $\left\{\mathrm{y}_{\mathrm{n}}\right\}$ also converges to the same limit z, i.e., $\lim _{n \rightarrow \infty} \operatorname{Px}_{2 n}=\lim _{n \rightarrow \infty} \operatorname{STx}_{2 n+1}=\lim _{n \rightarrow \infty} \mathrm{Qx}_{2 n+1}=$ $\lim _{\mathrm{n} \rightarrow \infty} \mathrm{ABx}_{2 \mathrm{n}+2}=\mathrm{z}$.

Case $\mathbf{I}$ ( $\mathrm{P}$ is continuous). In this case, we have

$\mathrm{PPx}_{2 \mathrm{n}} \rightarrow \mathrm{Pz}, \mathrm{P}(\mathrm{AB}) \mathrm{x}_{2 \mathrm{n}} \rightarrow \mathrm{Pz}$

The semi-compatibility of the pair $(\mathrm{P}, \mathrm{AB})$ gives

$\mathrm{P}(\mathrm{AB}) \mathrm{Px}_{2 \mathrm{n}}=\mathrm{ABz}$. As the limit of a sequence in N.M. Fuzzy metric Space is unique we have, $\mathrm{ABz}=\mathrm{Pz}$

Step I. By putting $\mathrm{x}=\mathrm{Px}_{2 \mathrm{n}}, \mathrm{y}=\mathrm{x}_{2 \mathrm{n}+1}$ in (3), we obtain that $\mathrm{g}\left(\mathrm{M}\left(\mathrm{PPx}_{2 \mathrm{n}}, \mathrm{Qx}_{2 \mathrm{n}+1}, \mathrm{t}\right)\right) \leq \phi\left(\max \left\{\mathrm{g}\left(\mathrm{M}\left((\mathrm{AB}) \mathrm{Px}_{2 \mathrm{n}}, \mathrm{ST}_{2 \mathrm{n}+1}, \mathrm{t}\right)\right)\right.\right.$,

$$
\mathrm{g}\left(\mathrm{M}\left((\mathrm{AB}) \mathrm{Px}_{2 \mathrm{n}} \mathrm{PPx}_{2 \mathrm{n}}, \mathrm{t}\right)\right), \mathrm{g}\left(\mathrm{M}\left(\mathrm{STx}_{2 \mathrm{n}+1}, \mathrm{Qx}_{2 \mathrm{n}+1}, \mathrm{t}\right)\right),
$$$$
\left.\left.1 / 2\left(\mathrm{~g}\left(\mathrm{M}\left((\mathrm{AB}) \mathrm{Px}_{2 \mathrm{n}}, \mathrm{Qx}_{2 \mathrm{n}+1}, \mathrm{t}\right)\right)+\mathrm{g}\left(\mathrm{M}\left(\mathrm{STx}_{2 \mathrm{n}+1}, \mathrm{PPx}_{2 \mathrm{n}}, \mathrm{t}\right)\right)\right)\right\}\right)
$$

Letting $n \rightarrow \infty$, we have

$\mathrm{g}(\mathrm{M}(\mathrm{Pz}, \mathrm{z}, \mathrm{t})) \leq \phi(\max \{\mathrm{g}(\mathrm{M}(\mathrm{Pz}, \mathrm{z}, \mathrm{t})), \mathrm{g}(\mathrm{M}(\mathrm{Pz}, \mathrm{Pz}, \mathrm{t}))$, $\mathrm{g}(\mathrm{M}(\mathrm{z}, \mathrm{z}, \mathrm{t})), 1 / 2(\mathrm{~g}(\mathrm{M}(\mathrm{Pz}, \mathrm{z}, \mathrm{t}))+\mathrm{g}(\mathrm{M}(\mathrm{z}, \mathrm{Pz}, \mathrm{t})))\})$

$\Rightarrow \mathrm{g}(\mathrm{M}(\mathrm{Pz}, \mathrm{z}, \mathrm{t})) \leq \phi(\max \{\mathrm{g}(\mathrm{M}(\mathrm{Pz}, \mathrm{z}, \mathrm{t})), 0,0$,

$$
\mathrm{g}(\mathrm{M}(\mathrm{Pz}, \mathrm{z}, \mathrm{t})))\})
$$

$\Rightarrow \mathrm{g}(\mathrm{M}(\mathrm{Pz}, \mathrm{z}, \mathrm{t})) \leq \phi\{\mathrm{g}(\mathrm{M}(\mathrm{Pz}, \mathrm{z}, \mathrm{t})\}$

$\Rightarrow \mathrm{g}(\mathrm{M}(\mathrm{Pz}, \mathrm{z}, \mathrm{t}))=0$.i.e., $\mathrm{M}(\mathrm{Pz}, \mathrm{z}, \mathrm{t})=1$

$\Rightarrow \mathrm{z}=\mathrm{Pz}$

Step II. As $P(X) \subset S T(X)$, there exists a point $w \in X$ such that $\mathrm{z}=\mathrm{Pz}=\mathrm{STw}$. By putting $\mathrm{x}=\mathrm{Px}_{2 \mathrm{n}}, \mathrm{y}=\mathrm{w}$ in (3), we obtain that

$\mathrm{g}\left(\mathrm{M}\left(\mathrm{PPx}_{2 \mathrm{n}}, \mathrm{Qw}, \mathrm{t}\right)\right) \leq \phi\left(\max \left\{\mathrm{g}\left(\mathrm{M}\left((\mathrm{AB}) \mathrm{Px}_{2 \mathrm{n}}, \mathrm{STw}, \mathrm{t}\right)\right)\right.\right.$, $\mathrm{g}\left(\mathrm{M}\left((\mathrm{AB}) \mathrm{Px}_{2 \mathrm{n}}, \mathrm{PPx}_{2 \mathrm{n}}, \mathrm{t}\right)\right), \mathrm{g}(\mathrm{M}(\mathrm{STw}, \mathrm{Qw}, \mathrm{t}))$, $\left.\left.1 / 2\left(\mathrm{~g}\left(\mathrm{M}\left((\mathrm{AB}) \mathrm{Px}_{2 \mathrm{n}}, \mathrm{Qw}, \mathrm{t}\right)\right)+\mathrm{g}\left(\mathrm{M}\left(\mathrm{STw}, \mathrm{PPx}_{2 \mathrm{n}}, \mathrm{t}\right)\right)\right)\right\}\right)$ Letting $\mathrm{n} \rightarrow \infty$, we have $\mathrm{g}(\mathrm{M}(\mathrm{z}, \mathrm{Qw}, \mathrm{t})) \leq \phi(\max \{\mathrm{g}(\mathrm{M}(\mathrm{z}, \mathrm{z}, \mathrm{t})), \mathrm{g}(\mathrm{M}(\mathrm{z}, \mathrm{z}, \mathrm{t}))$, $\mathrm{g}(\mathrm{M}(\mathrm{z}, \mathrm{Qw}, \mathrm{t})), 1 / 2(\mathrm{~g}(\mathrm{M}(\mathrm{z}, \mathrm{Qw}, \mathrm{t}))+\mathrm{g}(\mathrm{M}(\mathrm{z}, \mathrm{z}, \mathrm{t})))\})$ $\mathrm{g}(\mathrm{M}(\mathrm{z}, \mathrm{Qw}, \mathrm{t})) \leq \phi(\max \{0,0, \mathrm{~g}(\mathrm{M}(\mathrm{z}, \mathrm{Qw}, \mathrm{t}))$,

$$
1 / 2(\mathrm{~g}(\mathrm{M}(\mathrm{z}, \mathrm{Qw}, \mathrm{t})))\})
$$

$\Rightarrow \mathrm{g}(\mathrm{M}(\mathrm{z}, \mathrm{Qw}, \mathrm{t})) \leq \phi(\mathrm{g}(\mathrm{M}(\mathrm{z}, \mathrm{Qw}, \mathrm{t}))$

$\Rightarrow \mathrm{g}(\mathrm{M}(\mathrm{z}, \mathrm{Qw}, \mathrm{t}))=0$.i.e., $\mathrm{M}(\mathrm{z}, \mathrm{Qw}, \mathrm{t})=1$

$\Rightarrow \mathrm{z}=\mathrm{Qw}$.

Therefore $\mathrm{Qw}=\mathrm{STw}=\mathrm{z}$. Since $(\mathrm{Q}, \mathrm{ST})$ is weakly compatible, we get that

$(\mathrm{ST}) \mathrm{Q} \mathrm{w}=\mathrm{Q}(\mathrm{ST}) \mathrm{w}$, that is, $\mathrm{Qz}=\mathrm{STz}$.

Step III. By putting $\mathrm{x}=\mathrm{x}_{2 \mathrm{n}}, \mathrm{y}=\mathrm{z}$ in (3), we obtain that $\mathrm{g}\left(\mathrm{M}\left(\mathrm{Px}_{2 \mathrm{n}}, \mathrm{Qz}, \mathrm{t}\right)\right) \leq \phi\left(\max \left\{\mathrm{g}\left(\mathrm{M}\left(\mathrm{ABx}_{2 \mathrm{n}}, \mathrm{STz}, \mathrm{t}\right)\right)\right.\right.$,

$\mathrm{g}\left(\mathrm{M}\left(\mathrm{ABx}_{2 \mathrm{n}}, \mathrm{Px}_{2 \mathrm{n}}, \mathrm{t}\right)\right), \mathrm{g}(\mathrm{M}(\mathrm{STz}, \mathrm{Qz}, \mathrm{t}))$,

$\left.\left.1 / 2\left(\mathrm{~g}\left(\mathrm{M}\left(\mathrm{ABx}_{2 \mathrm{n}}, \mathrm{Qz}, \mathrm{t}\right)\right)+\mathrm{g}\left(\mathrm{M}\left(\mathrm{STz}, \mathrm{Px}_{2 \mathrm{n}}, \mathrm{t}\right)\right)\right)\right\}\right)$

$\mathrm{g}(\mathrm{M}(\mathrm{z}, \mathrm{Qz}, \mathrm{t})) \leq \phi(\max \{\mathrm{g}(\mathrm{M}(\mathrm{z}, \mathrm{Qz}, \mathrm{t})), \mathrm{g}(\mathrm{M}(\mathrm{z}, \mathrm{z}, \mathrm{t}))$, $\mathrm{g}(\mathrm{M}(\mathrm{Qz}, \mathrm{Qz}, \mathrm{t})), 1 / 2(\mathrm{~g}(\mathrm{M}(\mathrm{z}, \mathrm{Qz}, \mathrm{t}))+\mathrm{g}(\mathrm{M}(\mathrm{Qz}, \mathrm{z}, \mathrm{t})))\})$ $\mathrm{g}(\mathrm{M}(\mathrm{z}, \mathrm{Qz}, \mathrm{t})) \leq \phi(\max \{\mathrm{g}(\mathrm{M}(\mathrm{z}, \mathrm{Qz}, \mathrm{t})), 0,0, \mathrm{~g}(\mathrm{M}(\mathrm{z}, \mathrm{Qz}, \mathrm{t}))\})$ $\Rightarrow \mathrm{g}(\mathrm{M}(\mathrm{z}, \mathrm{Qz}, \mathrm{t})) \leq \phi(\mathrm{g}(\mathrm{M}(\mathrm{z}, \mathrm{Qz}, \mathrm{t}))$ $\Rightarrow \mathrm{g}(\mathrm{M}(\mathrm{z}, \mathrm{Qz}, \mathrm{t}))=0$.i.e., $\mathrm{M}(\mathrm{z}, \mathrm{Qz}, \mathrm{t})=1$ $\Rightarrow \mathrm{z}=\mathrm{Qz}$.

Step IV. Putting $\mathrm{x}=\mathrm{x}_{2 \mathrm{n}}, \mathrm{y}=\mathrm{Tz}$ in (3) we get, $\mathrm{g}\left(\mathrm{M}\left(\mathrm{Px}_{2 \mathrm{n}}, \mathrm{QTz}, \mathrm{t}\right)\right) \leq \phi\left(\max \left\{\mathrm{g}\left(\mathrm{M}\left(\mathrm{ABx}_{2 \mathrm{n}}, \mathrm{STTz}, \mathrm{t}\right)\right)\right.\right.$, 


$$
\begin{gathered}
\mathrm{g}\left(\mathrm{M}\left(\mathrm{ABx}_{2 \mathrm{n}}, \mathrm{Px}_{2 \mathrm{n}}, \mathrm{t}\right)\right), \mathrm{g}(\mathrm{M}(\mathrm{STTz}, \mathrm{QTz}, \mathrm{t})), \\
\left.\left.1 / 2\left(\mathrm{~g}\left(\mathrm{M}\left(\mathrm{ABx}_{2 \mathrm{n}}, \mathrm{QTz}, \mathrm{t}\right)\right)+\mathrm{g}\left(\mathrm{M}\left(\mathrm{STT}_{\mathrm{N}}, \mathrm{Px}_{2 \mathrm{n}}, \mathrm{t}\right)\right)\right)\right\}\right)
\end{gathered}
$$

As $\mathrm{QT}=\mathrm{TQ}$ and $\mathrm{ST}=\mathrm{TS}$ we have $\mathrm{QTz}=\mathrm{TQz}=\mathrm{Tz}$ and $\mathrm{ST}(\mathrm{Tz})=\mathrm{T}(\mathrm{ST}) \mathrm{z}=\mathrm{Tz}$.

Letting $\mathrm{n} \rightarrow \infty$ we get,

$\mathrm{g}(\mathrm{M}(\mathrm{z}, \mathrm{Tz}, \mathrm{t})) \leq \phi(\max \{\mathrm{g}(\mathrm{M}(\mathrm{z}, \mathrm{Tz}, \mathrm{t})), \mathrm{g}(\mathrm{M}(\mathrm{z}, \mathrm{z}, \mathrm{t}))$,

$\mathrm{g}(\mathrm{M}(\mathrm{Tz}, \mathrm{Tz}, \mathrm{t})), 1 / 2(\mathrm{~g}(\mathrm{M}(\mathrm{z}, \mathrm{Tz}, \mathrm{t}))+\mathrm{g}(\mathrm{M}(\mathrm{Tz}, \mathrm{z}, \mathrm{t})))\})$

$\mathrm{g}(\mathrm{M}(\mathrm{z}, \mathrm{Tz}, \mathrm{t})) \leq \phi(\max \{\mathrm{g}(\mathrm{M}(\mathrm{z}, \mathrm{Tz}, \mathrm{t})), 0,0, \mathrm{~g}(\mathrm{M}(\mathrm{z}, \mathrm{Tz}, \mathrm{t}))\})$

$\Rightarrow \mathrm{g}(\mathrm{M}(\mathrm{z}, \mathrm{Tz}, \mathrm{t})) \leq \phi(\mathrm{g}(\mathrm{M}(\mathrm{z}, \mathrm{Tz}, \mathrm{t}))$

$\Rightarrow \mathrm{g}(\mathrm{M}(\mathrm{z}, \mathrm{Tz}, \mathrm{t}))=0$.i.e., $\mathrm{M}(\mathrm{z}, \mathrm{Tz}, \mathrm{t})=1$

$\Rightarrow \mathrm{z}=\mathrm{Tz}$

Now $\mathrm{STz}=\mathrm{Tz}=\mathrm{z}$ implies $\mathrm{Sz}=\mathrm{z}$. Hence $\mathrm{Sz}=\mathrm{Tz}=\mathrm{Qz}=\mathrm{z}$.

Step V. By putting $x=B z, y=x_{2 n+1}$ in (3), we obtain that

$\mathrm{g}\left(\mathrm{M}\left(\mathrm{PBz}, \mathrm{Qx}_{2 \mathrm{n}+1}, \mathrm{t}\right)\right) \leq \phi\left(\max \left\{\mathrm{g}\left(\mathrm{M}\left((\mathrm{AB}) \mathrm{Bz}, \mathrm{STx}_{2 \mathrm{n}+1}, \mathrm{t}\right)\right)\right.\right.$,

$\mathrm{g}(\mathrm{M}((\mathrm{AB}) \mathrm{Bz}, \mathrm{PBz}, \mathrm{t})), \mathrm{g}\left(\mathrm{M}\left(\mathrm{STx}_{2 \mathrm{n}+1}, \mathrm{Qx}_{2 \mathrm{n}+1}, \mathrm{t}\right)\right)$,

$\left.\left.1 / 2\left(g\left(M\left((A B) B z, Q x_{2 n+1}, t\right)\right)+g\left(M\left(\operatorname{STx}_{2 n+1}, P B z, t\right)\right)\right)\right\}\right)$

As $\mathrm{BP}=\mathrm{PB}, \mathrm{AB}=\mathrm{BA}$ so we have $\mathrm{P}(\mathrm{Bz})=\mathrm{B}(\mathrm{Pz})=\mathrm{Bz}$ and

$\mathrm{AB}(\mathrm{Bz})=\mathrm{B}(\mathrm{ABz})=\mathrm{Bz}$.

Letting $\mathrm{n} \rightarrow \infty$, we have

$\mathrm{g}(\mathrm{M}(\mathrm{Bz}, \mathrm{z}, \mathrm{t})) \leq \phi(\max \{\mathrm{g}(\mathrm{M}(\mathrm{Bz}, \mathrm{z}, \mathrm{t})), \mathrm{g}(\mathrm{M}(\mathrm{Bz}, \mathrm{Bz}, \mathrm{t}))$,

$\mathrm{g}(\mathrm{M}(\mathrm{z}, \mathrm{z}, \mathrm{t})), 1 / 2(\mathrm{~g}(\mathrm{M}(\mathrm{Bz}, \mathrm{z}, \mathrm{t}))+\mathrm{g}(\mathrm{M}(\mathrm{z}, \mathrm{Bz}, \mathrm{t})))\})$

$\Rightarrow \mathrm{g}(\mathrm{M}(\mathrm{Bz}, \mathrm{z}, \mathrm{t})) \leq \phi(\max \{\mathrm{g}(\mathrm{M}(\mathrm{Bz}, \mathrm{z}, \mathrm{t})), 0,0$,

$$
\mathrm{g}(\mathrm{M}(\mathrm{Bz}, \mathrm{z}, \mathrm{t})))\})
$$

$\Rightarrow \mathrm{g}(\mathrm{M}(\mathrm{Bz}, \mathrm{z}, \mathrm{t})) \leq \phi\{\mathrm{g}(\mathrm{M}(\mathrm{Bz}, \mathrm{z}, \mathrm{t})\}$

$\Rightarrow \mathrm{g}(\mathrm{M}(\mathrm{Bz}, \mathrm{z}, \mathrm{t}))=0$.i.e., $\mathrm{M}(\mathrm{Bz}, \mathrm{z}, \mathrm{t})=1$

$\Rightarrow \mathrm{z}=\mathrm{Bz}$ and $\mathrm{ABz}=\mathrm{z}$ implies $\mathrm{Az}=\mathrm{z}$. Therefore, $\mathrm{Az}=\mathrm{Bz}=$ $\mathrm{Pz}=\mathrm{z}$.

Hence we have $\mathrm{Az}=\mathrm{Bz}=\mathrm{Pz}=\mathrm{Sz}=\mathrm{Tz}=\mathrm{Qz}=\mathrm{z}$ that is, $\mathrm{z}$ is a common fixed point of $\mathrm{A}, \mathrm{B}, \mathrm{S}, \mathrm{T}, \mathrm{P}$ and $\mathrm{Q}$.

Case II ( $\mathrm{AB}$ is continuous). In this case, we have

$(\mathrm{AB})^{2} \mathrm{x}_{2 \mathrm{n}} \rightarrow \mathrm{ABz}$ and $(\mathrm{AB}) \mathrm{Px}_{2 \mathrm{n}} \rightarrow \mathrm{ABz}$

As $(\mathrm{P}, \mathrm{AB})$ is semicompatible, we have $\mathrm{P}(\mathrm{AB}) \mathrm{x}_{2 \mathrm{n}} \rightarrow \mathrm{ABz}$.

Step VI. By putting $\mathrm{x}=\mathrm{ABx}_{2 \mathrm{n}}, \mathrm{y}=\mathrm{x}_{2 \mathrm{n}+1}$ in (3), we obtain that $\mathrm{g}\left(\mathrm{M}\left(\mathrm{PABx}_{2 \mathrm{n}}, \mathrm{Qx}_{2 \mathrm{n}+1}, \mathrm{t}\right)\right) \leq \phi\left(\max \left\{\mathrm{g}\left(\mathrm{M}\left(\mathrm{ABABx}_{2 \mathrm{n}}, \mathrm{STx}_{2 \mathrm{n}+1}\right.\right.\right.\right.$, t)), $\mathrm{g}\left(\mathrm{M}\left(\mathrm{ABABx}_{2 \mathrm{n}}, \mathrm{PABx}_{2 \mathrm{n}}, \mathrm{t}\right)\right), \mathrm{g}\left(\mathrm{M}\left(\mathrm{STx}_{2 \mathrm{n}+1}, \mathrm{Qx}_{2 \mathrm{n}+1}, \mathrm{t}\right)\right)$,

$1 / 2\left(\mathrm{~g}\left(\mathrm{M}\left(\mathrm{ABABx}_{2 \mathrm{n}}, \mathrm{Qx}_{2 \mathrm{n}+1}, \mathrm{t}\right)\right)+\right.$

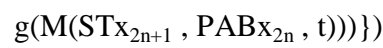

Letting $\mathrm{n} \rightarrow \infty$, we have

$\mathrm{g}(\mathrm{M}(\mathrm{ABz}, \mathrm{z}, \mathrm{t})) \leq \phi(\max \{\mathrm{g}(\mathrm{M}(\mathrm{ABz}, \mathrm{z}, \mathrm{t}))$,
$\mathrm{g}(\mathrm{M}(\mathrm{ABz}, \mathrm{ABz}, \mathrm{t})), \mathrm{g}(\mathrm{M}(\mathrm{z}, \mathrm{z}, \mathrm{t}))$,

$1 / 2(\mathrm{~g}(\mathrm{M}(\mathrm{ABz}, \mathrm{z}, \mathrm{t}))+\mathrm{g}(\mathrm{M}(\mathrm{z}, \mathrm{ABz}, \mathrm{t})))\})$

$\Rightarrow \mathrm{g}(\mathrm{M}(\mathrm{ABz}, \mathrm{z}, \mathrm{t})) \leq \phi(\max \{\mathrm{g}(\mathrm{M}(\mathrm{ABz}, \mathrm{z}, \mathrm{t})), 0,0,(\mathrm{~g}(\mathrm{M}(\mathrm{z}$ $\mathrm{ABz}, \mathrm{t})))\})$

$\Rightarrow \mathrm{g}(\mathrm{M}(\mathrm{ABz}, \mathrm{z}, \mathrm{t})) \leq \phi\{\mathrm{g}(\mathrm{M}(\mathrm{ABz}, \mathrm{z}, \mathrm{t})\}$

$\Rightarrow \mathrm{g}(\mathrm{M}(\mathrm{ABz}, \mathrm{z}, \mathrm{t}))=0$.i.e., $\mathrm{M}(\mathrm{ABz}, \mathrm{z}, \mathrm{t})=1$

$\Rightarrow \mathrm{z}=\mathrm{ABz}$ and rest of the proof follows from Step III onwards of the previous case.

Uniqueness. Let $\mathrm{u}$ be another common fixed point of A, B, S, T, P and Q.

Then $\mathrm{u}=\mathrm{Au}=\mathrm{Su}=\mathrm{Bu}=\mathrm{Tu}=\mathrm{Pu}=\mathrm{Qu}$.

Putting $\mathrm{x}=\mathrm{z}$ and $\mathrm{y}=\mathrm{u}$ in (3), we have

$\mathrm{g}(\mathrm{M}(\mathrm{Pz}, \mathrm{Qu}, \mathrm{t})) \leq \phi(\max \{\mathrm{g}(\mathrm{M}(\mathrm{ABz}, \mathrm{STu}, \mathrm{t})), \mathrm{g}(\mathrm{M}(\mathrm{ABz}$,

$\mathrm{Pz}, \mathrm{t})), \mathrm{g}(\mathrm{M}(\mathrm{STu}, \mathrm{Qu}, \mathrm{t}))$,

$1 / 2(\mathrm{~g}(\mathrm{M}(\mathrm{ABz}, \mathrm{Qu}, \mathrm{t}))+\mathrm{g}(\mathrm{M}(\mathrm{STu}, \mathrm{Pz}, \mathrm{t})))\})$,

$\Rightarrow \mathrm{g}(\mathrm{M}(\mathrm{z}, \mathrm{u}, \mathrm{t})) \leq \phi(\max \{\mathrm{g}(\mathrm{M}(\mathrm{z}, \mathrm{u}, \mathrm{t})), \mathrm{g}(\mathrm{M}(\mathrm{z}, \mathrm{z}, \mathrm{t})), \mathrm{g}(\mathrm{M}(\mathrm{u}$, $\mathrm{u}, \mathrm{t})), 1 / 2(\mathrm{~g}(\mathrm{M}(\mathrm{z}, \mathrm{u}, \mathrm{t}))+\mathrm{g}(\mathrm{M}(\mathrm{u}, \mathrm{z}, \mathrm{t})))\})$,

$\Rightarrow \mathrm{g}(\mathrm{M}(\mathrm{z}, \mathrm{u}, \mathrm{t})) \leq \phi(\max \{\mathrm{g}(\mathrm{M}(\mathrm{z}, \mathrm{u}, \mathrm{t})), 0,0, \mathrm{~g}(\mathrm{M}(\mathrm{z}, \mathrm{u}, \mathrm{t}))\})$,

$\Rightarrow \mathrm{g}(\mathrm{M}(\mathrm{z}, \mathrm{u}, \mathrm{t})) \leq \phi\{\mathrm{g}(\mathrm{M}(\mathrm{z}, \mathrm{u}, \mathrm{t})\}$

$\Rightarrow \mathrm{g}(\mathrm{M}(\mathrm{z}, \mathrm{u}, \mathrm{t}))=0$.i.e., $\mathrm{M}(\mathrm{z}, \mathrm{u}, \mathrm{t})=1$

$\Rightarrow \mathrm{z}=\mathrm{u}$

Therefore, $\mathrm{z}$ is the unique common fixed point of the selfmaps A, B, S, T, P and Q.

Corollary 1.1. Let A, B, S , T $: X \rightarrow X$ be mappings such that

(1) $\mathrm{A}(\mathrm{X}) \subset \mathrm{T}(\mathrm{X})$ and $\mathrm{B}(\mathrm{X}) \subset \mathrm{S}(\mathrm{X})$,

(2) $\mathrm{g}(\mathrm{M}(\mathrm{Ax}, \mathrm{By}, \mathrm{t})) \leq \phi(\max \{\mathrm{g}(\mathrm{M}(\mathrm{Sx}, \mathrm{Ty}, \mathrm{t}))$ $\mathrm{g}(\mathrm{M}(\mathrm{Sx}, \mathrm{Ax}, \mathrm{t})), \mathrm{g}(\mathrm{M}(\mathrm{Ty}, \mathrm{By}, \mathrm{t}))$,

$1 / 2(g(M(S x, B y, t))+g(M(T y, A x, t)))\})$ for all $t>0$,

where a function $\phi:[0, \infty) \rightarrow[0, \infty)$ satisfies the condition $(\Phi)$.

(3) the pair $(\mathrm{A}, \mathrm{S})$ is semicompatible and $(\mathrm{B}, \mathrm{T})$ is weakly compatible.

(4) one of A or S is continuous

Then $\mathrm{A}, \mathrm{B}, \mathrm{S}$ and $\mathrm{T}$ have a uniqe common fixed point in $\mathrm{X}$.

Proof. As semicompatibility implies weak compatibility, the proof follows from theorem 1.1.

Now, taking $\mathrm{S}=\mathrm{I}$ and $\mathrm{T}=\mathrm{I}$ in theorem in 1.1 , we get the following corollary.

Corollary 1.2. Let $A, B: X \rightarrow X$ be mappings such that

(1) $\mathrm{g}(\mathrm{M}(\mathrm{Ax}, \mathrm{By}, \mathrm{t})) \leq \phi(\max \{\mathrm{g}(\mathrm{M}(\mathrm{x}, \mathrm{y}, \mathrm{t}))$,

$\mathrm{g}(\mathrm{M}(\mathrm{x}, \mathrm{Ax}, \mathrm{t})), \mathrm{g}(\mathrm{M}(\mathrm{y}, \mathrm{By}, \mathrm{t}))$, 
$1 / 2(\mathrm{~g}(\mathrm{M}(\mathrm{x}, \mathrm{By}, \mathrm{t}))+\mathrm{g}(\mathrm{M}(\mathrm{y}, \mathrm{Ax}, \mathrm{t})))\})$ for all $\mathrm{t}>0$, where a function $\phi:[0, \infty) \rightarrow[0, \infty)$ satisfies the condition $(\Phi)$.

Then $\mathrm{A}, \mathrm{B}$ have a unique common fixed point in $\mathrm{X}$.

\section{CONCLUSION}

In this work we have dealt with fixed points of six mappings in which one pair of three mappings is semicompatible and other pair of three mappings is weakcompatible. The future scope is to obtained quartet fixed points for mappings in this space.

\section{ACKNOWLEDGMENTS}

Our thanks to the referees for their helpful and valuable comments.

\section{REFERENCES}

[1] A. George and P. Veeramani, 1994. On some results in fuzzy metric space, Fuzzy Sets and Systems, vol. 64, 395-399.

[2] B. Schweizer, H. Sherwood, and R. M. Tardi ${ }^{\circledR}, 1988$. Contractions on PMspace examples and counter examples, Stochastica vol. 1, 5-17.

[3] D. Mihet, 2004. A Banach contraction theorem in fuzzy metric spaces, Fuzzy Sets and Systems, vol. 144, 431439.

[4] D. Mihet, 2008. Fuzzy $\psi$-contractive mappings in non-Archimedean fuzzy metric spaces, Fuzzy Sets and Systems vol. 159, $739-744$.

[5] G. L. Cain Jr. and R. H. Kasreil, 1976. Fixed and periodic points of local contraction mappings on probabilistic metric spaces, Math. Systems Theory, vol. 9, 289-297.

[6] I. Kramosil and J. Michalek, 1975. Fuzzy metric and statistical metric spaces, Kybernetica, vol. 11, 326-334.
[7] I. Istratescu, 1985. On some fixed point theorems for mappings on non-Archimedean probabilistic metric spaces, Seminarual de Teoria probabilistic tatiler si. applicatii.,vol. 73, 1-11.

[8] L.A. Zadeh, 1965. Fuzzy Sets, Information and Control, vol. 8, 338-353.

[9] L.A. Zadeh, 1969. Biological application of the theory of fuzzy sets and systems, in: Proc. Int. Symp. Biocybernetics of the Central Nerous System (Little, Brown \& Co., Boston) 199-212.

[10] M. S. El Naschie, 1998. On the uncertainty of Cantorian geometry and two-slit experiment,Chaos, Solitons and Fractals vol. 9, 517-529.

[11] M. S. El Naschie, 2004. A review of E-infinity theory and the mass spectrum of high energy particle physics, Chaos, Solitons and Fractals vol. 19, 209-236.

[12] M. S. El Naschie, 2005. On a fuzzy Kahler-like Manifold which is consistent with two-slit experiment, Int. J. of Nonlinear Science and Numerical Simulation, vol. 6, 95-98.

[13] V. Gregori and A. Sapena, 2002. On fixed-point theorem in fuzzy metric spaces, Fuzzy Sets and Systems, vol. $125,245-252$.

[14] Y. Tanaka, Y. Mizno, and T. Kado, 2005. Chaotic dynamics in Friedmann equation, Chaos, Solitons and Fractals vol. 24, 407-422.

[15] S. S. Chang, 1985. On the theory of probabilistic metric spaces with applications, Acta Math. Sinica, New Series, vol. 1 , issue $4,366-377$.

[16] S. S. Chang, 1983. Fixed point theorems for generalized Meir-Keeler Type mappings, J. Sichuan Univ., Natural Sci. Edition, vol. 2, 17-23. 